\title{
Haplotype Diversity of Swamp Buffalo and River Buffalo Based on Cytochrome B Gene: A Study of Meta-Analysis
}

\author{
F. Saputra ${ }^{\mathrm{a}, *}$, A. Anggraeni ${ }^{\mathrm{a}}$, A. B. L. Ishak ${ }^{\mathrm{a}}$, A. Hafid ${ }^{\mathrm{a}}$, M. Rusdin ${ }^{\mathrm{b}}$, \& C. Sumantri ${ }^{\mathrm{c}}$ \\ andonesian Research Institute for Animal Production \\ Jalan Veteran III, Bogor 16720, Indonesia \\ bepartment of Animal Science, Faculty of Animal Science, Halu Oleo University \\ Kampus Hijau Bumi Tridharma, Jalan H.E.A. Mokodompit, Anduonohu, Kendari, Sulawesi Tenggara 93232, Indonesia \\ 'Department of Animal Production and Technology, Faculty of Animal Science, IPB University \\ Jalan Agatis, Kampus IPB Darmaga Bogor 16680, Indonesia \\ *Corresponding author: ferdysaputra@pertanian.go.id; ferdy44saputra@gmail.com \\ (Received 03-10-2020; Revised 17-04-2021; Accepted 03-05-2021)
}

\begin{abstract}
Buffalo (Bubalus bubalis) is well known as a domesticated buffalo in Asia. The genetic diversity of buffaloes in Asia needs to be studied to ensure a proper breeding program. A meta-analysis study on the cytochrome $b$ gene of the mitochondrial genome from various published data was conducted to evaluate genetic variation and haplo-geography of Asian buffaloes. A meta-analysis is used to provide a comprehensive view of the data. A total of 1369 swamp buffaloes Cytochrome B sequences (from Indonesia (79), Bangladesh (98), China (909), India (4), Laos (96), Taiwan (29), Thailand (54), and Vietnam (100)) and 91 river buffaloes (from China (42), Nepal (42), and Pakistan (7)) were used in this study. Cytochrome B sequences $(678 \mathrm{bp})$ of Syncerus caffer, Bubalus arnee, Bubalus depressicornis, Bubalus quarlesi, Bubalus mindorensis, swamp buffalo, and river buffalo were determined for their haplotypes using DnaSP v6 program. Haplotypes were analyzed by Principal Coordinate Analysis (PCoA) using Adegenet Package and Hierarchical Clustering on Principal Components (HCPC) methods using Factoextra and FactoMineR Package in R-4.0.0 program. Bayesian analysis of genetic differentiation was implemented in BAPS 6.0. Furthermore, we found 56 haplotypes for swamp buffaloes in eight Asian countries and 5 haplotypes for river buffaloes in Pakistan. We also found 5 haplotypes for outgroup ( $B$. arnee, S. caffer, B. depressicornis, B. quarlesi, B. mindorensis). Therefore, we found 66 haplotypes in total with outgroup sequences. Based on the PCoA results, three clusters were found. However, the HCPC found eight clusters. Based on HCPC, countries in East and South Asia have four maternal lineages. This is evidence that buffalo domestication has first occurred in East-South Asia. In conclusion, we found four maternal lineages of swamp buffalo and two maternal lineages of river buffalo from ten countries. We also found one maternal lineage for Syncerus caffer and one maternal lineage for B. depressicornis, $B$. quarlesi, and B. mindorensis.
\end{abstract}

Keywords: haplotype diversity; swamp buffalo; cytochrome b; meta-analysis

\section{INTRODUCTION}

Buffaloes are commonly used as livestock for producing meat and milk. However, buffaloes are still used as draught animals and have not been kept intensively like cattle and chickens. In Indonesia, swamp and river buffaloes get less concern than cows and chickens, which are strengthened by better research funding. Genetic diversity studies are needed to determine a good breeding program for buffaloes in the future.

Evaluation of genetic variability and genetic association in populations is essential to control the loss of genetic diversity for selective breeding (MastrochiricoFilho et al., 2019). Breeding programs can be implemented if genetic diversity is observed and maintained. In the same way, genomic tools will allow breeding strategies to ensure the improvement of performance and preserve genetic diversity (Taberlet et al., 2011).

Genetic diversity in livestock is also useful for distinguishing between populations. To find out genetic differences between populations, phenotypic traits are generally used or based on gene diversity. Sequence data have been widely known to observe relationships between organisms (Konishi et al., 2019). To identify variations in the maternal lineage, the mitochondrial genomes are usually explored. In the mitochondrial genome, there are D-loop, Cytochrome b gene (Cyt B), and Cytochrome Oxidase I (COI) gene markers that are often used to evaluate kinship (Kumar et al., 2007; Saputra et al. 2013; Paraguas et al., 2018). Research conducted by Tobe et al. (2010) has shown that the diversity of Cyt B is higher than that of COI. Meta-analysis might contribute 
to a better understanding of the domestication history of animals (Guangxin et al., 2016). A recent study of cytochrome b in swamp buffalo by Sun et al. (2020) showed 5 maternal lineages of swamp buffalo using neighborjoining in six countries (China, Thailand, Vietnam, Laos, India, and Bangladesh). Kumar et al. (2007) suggested that swamp and river buffaloes were domesticated independently and the classification of swamp and river buffaloes as two related subspecies is more suitable. The evaluation of the genetic diversity of cytochrome B in swamp and river buffaloes has been widely carried out. However, a meta-analysis study has never been carried out to analyze the genetic diversity of swamp buffalo in Asia. Therefore, we identified cytochrome B variation in swamp and river buffaloes from various published data in this meta-analysis study.

\section{MATERIALS AND METHODS}

A total of 1369 swamp buffalo sequences (originating from Bangladesh, China, India, Indonesia, Laos, Taiwan, Thailand, Vietnam), 91 river buffalo sequences (originating from China, Nepal, and Pakistan), and five outgroup sequences (Bubalus arnee, Bubalus depressicornis, Bubalus quarlesi, Bubalus mindorensis, and Syncerus caffer) were used in this study. The data used in this study are published data (Table 1). The data used were base positions 70 to 747 (678 bp) of Cytochrome B gene based on GenBank reference (D82894). We used those base positions to accommodate the length of cytochrome $B$ gene sequence of swamp buffalo from Indonesia based on the result reported by Rusdin et al. (2020). Sequences data were analyzed using the DnaSP v6 program to determine the haplotypes and haplotype diversity (Rozas et al., 2017). The haplotype data were further analyzed using the Principal Coordinate Analysis (PCoA) using the Adegenet Package (Jombart, 2008) in the R-4.0.0 program ( $\mathrm{R}$ Core, 2020). Furthermore, Hierarchical Clustering on Principal Components (HCPC) was drawn using Factoextra (Kassambara \& Mundt, 2020) and FactoMineR (Husson et al., 2015) packages from p-distance data in MEGA 7.0.26 (Kumar et al., 2016). Bayesian analysis of genetic differentiation was implemented in BAPS 6.0 (Cheng et al., 2013).

\section{RESULTS}

Based on sequences used in this study, Indonesian buffaloes with 80 sequences have a diversity of haplotypes of 0.6503 (Table 2). In 10 countries, we found

Table 1. Number of sequences from public database

\begin{tabular}{|c|c|c|c|c|}
\hline Accession number & Organism & Country & Total (n) & References \\
\hline D32193 & Bubalus arnee bubalis & - & 1 & (Chikuni et al., 1995) \\
\hline D82888 & Syncerus caffer & & 1 & (Tanaka et al., 1996) \\
\hline D82890 & Bubalus depressicornis & Indonesia & 1 & (Tanaka et al., 1996) \\
\hline D82891 & Bubalus quarlesi & Indonesia & 1 & (Tanaka et al., 1996) \\
\hline D82895 & Bubalus mindorensis & Philippines & 1 & (Tanaka et al., 1996) \\
\hline D82894 & Bubalus bubalis & Indonesia & 1 & (Tanaka et al., 1996) \\
\hline $\begin{array}{l}\text { JF946519, JF946520, JF946521, JF946522, JF946523, } \\
\text { JF946524, JF946525 }\end{array}$ & Bubalus bubalis (River buffalo) & Pakistan & 7 & (Saif et al., 2012) \\
\hline FJ467648 - FJ467917 & Bubalus bubalis & China & 270 & (Lei et al., 2011) \\
\hline EF409939, EF409940, EF409941, EF409942 & Bubalus bubalis & India & 4 & (Kumar et al., 2007) \\
\hline KR010069-KR010168 & Bubalus bubalis & Vietnam & 100 & (Zhang et al., 2016) \\
\hline KR010040-KR010068 & Bubalus bubalis & Taiwan & 29 & (Zhang et al., 2016) \\
\hline KR009986- KR010039 & Bubalus bubalis & Thailand & 54 & (Zhang et al., 2016) \\
\hline KR009944-KR009985 & Bubalus bubalis (River buffalo) & Nepal & 42 & (Zhang et al., 2016) \\
\hline KR009848-KR009943 & Bubalus bubalis & Laos & 96 & (Zhang et al., 2016) \\
\hline KR009167-KR009644; KR009687-KR009847 & Bubalus bubalis & China & 639 & (Zhang et al., 2016) \\
\hline KR009645-KR009666 & $\begin{array}{l}\text { Bubalus bubalis (River buffalo) } \\
\text { (Murrah) }\end{array}$ & China & 22 & (Zhang et al., 2016) \\
\hline KR009667-KR009686 & $\begin{array}{l}\text { Bubalus bubalis (River buffalo) } \\
\text { (Nili Ravi) }\end{array}$ & China & 20 & (Zhang et al., 2016) \\
\hline KR009069-KR009166 & Bubalus bubalis & Bangladesh & 98 & (Zhang et al., 2016) \\
\hline $\begin{array}{l}\text { BK6, BK7, BK8, BK9, BK12, BK14, BK15, BK18, } \\
\text { BK21, BK24, BK28, BD1, BD4, BD7, BD9, BD11, } \\
\text { BD13, BD14, BD15, BD19, BD25, KL1, KL2, KL3 } \\
\text { KL4, KL5, KL6, KL7, KL8, KL9, KL10, KL11, KLB1, } \\
\text { KLB2, KN1, KN2, KN3, KN4, KN5, KN6, KN7, } \\
\text { KN8, KN9, KN10, KN11, KN12, KN13, KN14, } \\
\text { TRB1, TRB2, TRB3, TRB4, TRB5, TRP6, TRP8, } \\
\text { TRT13, TRT14, TRT15, NTB1, NTB2, NTB3, NTB5, } \\
\text { NTB6, NTB7, NTB8, NTB9, NTB10, NTB11, BTN1, } \\
\text { BTN2, BTN3, BTN4, BTN5, NAD1, NAD2, NAD3, } \\
\text { NAD9, NAD10 }\end{array}$ & Bubalus bubalis & Indonesia & 78 & (Rusdin et al., 2020) \\
\hline
\end{tabular}


61 haplotypes, and the haplotype diversity was 0.7020 with 62 polymorphic sites. The smallest haplotype diversity was found in Taiwan (0.1970), and the highest was found in India (1.0000). The highest number of haplotypes was located in China (36 haplotypes), and the lowest number of haplotypes was found in Nepal (3 haplotypes) and Taiwan (3 haplotypes). By using data of S. caffer, B. arnee, B. quarlesi, B. mindorensis, and B. depressicornis, we found 66 haplotypes (Table 3). Haplotype 16 was the most common with large samples, i.e., $73.50 \%$ in China, $7.31 \%$ in Vietnam, $5.85 \%$ in Laos, $5.05 \%$ in Indonesia, $3.47 \%$ in Taiwan, $3.47 \%$ in Thailand, and $1.45 \%$ in Bangladesh (Table 4 ). We also found unique haplotypes in Bangladesh (6), China (24), India (1), Indonesia (8), Laos (1), Thailand (3), Pakistan (5), and Vietnam (1). Based on PCoA results, we found three clusters (Figure 1). The first cluster consisted of buffaloes from B. depressicornis, Bangladesh, China, India, Nepal, and Pakistan. The second cluster included buffaloes from B. arnee, B. quarlesi, B. mindorensis, Bangladesh, China, Indonesia, Laos, Nepal, Taiwan, Thailand, and Vietnam. On the other hand, S. caffer was very far from the other clusters.

HCPC showed eight clusters (Figure 2), i.e., cluster I: consisted of buffaloes from $B$. arnee, Bangladesh China, Indonesia, Laos, Nepal, Taiwan, Thailand, and Vietnam; cluster II: consisted of buffaloes from Bangladesh, China, India, Indonesia, Laos, Nepal, Taiwan, Thailand, and Vietnam; cluster III: consisted of buffaloes from Bangladesh, China, India, Nepal, and Pakistan; cluster IV: consisted of buffaloes from Bangladesh, China, India, Nepal, and Pakistan; cluster $\mathrm{V}$ : consisted of buffaloes from Pakistan; cluster VI: consisted of buffaloes from Pakistan; cluster VII: consisted of buffaloes from B. mindorensis, B. quarlesi, and $B$. depressicornis; cluster VIII: consisted of buffaloes from S. caffer. According to HCPC results, swamp buffaloes in Asia have four maternal lineages. Interestingly, haplotypes from Pakistan form separate clusters (cluster $\mathrm{V}$ and VI) for river buffalo. Indonesia, Laos, Taiwan, Thailand, and Vietnam only have two maternal lineages. Indian buffalo has three maternal lineages. On the other hand, Bangladesh, China, Nepal, and Pakistan have four maternal lineages. What is interesting is that the four maternal lineages are found in East and South Asian countries. Bayesian analysis showed 3 clusters (Figure
3) that were very similar to the results shown by the principal coordinate analysis. Interestingly, we found sequences of river buffaloes from Nepal and China in haplotypes 22 and 23 along with sequences from swamp buffaloes. This result is possible because we only took $678 \mathrm{bp}$ to observe the genetic diversity of the buffalo in this study.

\section{DISCUSSION}

Haplotype diversity based on the mtDNA of Egyptian and Indian buffaloes ranged from 0.8236 \pm 0.0488 to $0.9428 \pm 0.0088$ (Nagarajan et al., 2015). Lei et al. (2011) found that the haplotype diversity of Chinese buffaloes ranged from $0.469 \pm 0.039$ to $0.815 \pm$ 0.033 . Based on this haplotype diversity, Taiwan buffalo has lower haplotype diversity than buffaloes in the other countries. However, the gene diversity based on Cytochrome B in Indonesian buffalo is more diverse than the other studies on GH, GHR, GHRH, Pit1, COI, and microsatellite (Andreas et al., 2010; Misrianti et al., 2010; Saputra et al., 2013, 2020; Sumantri et al., 2010). The results we found with the HCPC showed that the haplotypes of B. depressicornis (Lowland Anoa), B. quarlesi (Mountain Anoa), and B. mindorensis (Tamaraw) were self-clustered, and our findings are different from result reported by Tanaka et al. (1996) that B. mindorensis is genetically closer to swamp buffalo than to anoa.

Data of B. arnee (Wild water buffalo) were obtained from Chikuni et al. (1995), showing a genetic closeness with swamp buffalo. Zhang et al. (2020) suggest the swamp and river buffaloes are descended from distinct populations of wild water buffalo (B. arnee). The river buffaloes originating from Pakistan have different haplotypes from the river buffaloes originating from China. Of the seven haplotypes of the Pakistani river buffalo (Saif et al., 2012), only five haplotypes are unique in this meta-analysis. Most likely, the domestication of river buffalo occurs in South Asia. Youssef et al. (2021) found three maternal lineages for river buffaloes in five countries (Bangladesh, China, Egypt, India, and Italy) based on the mitochondrial genome. The study also found that Indian river buffaloes had more maternal lineage. Nagarajan et al. (2015) suggested that the river buffalo was first domesticated in the Northwestern region of India and spread to other parts of the world. In other

Table 2. Haplotype diversity of buffaloes based on country

\begin{tabular}{lcccc}
\hline Population & $\mathrm{N}$ & Number of haplotypes & Variable sites & Haplotype diversity \\
\hline Pakistan & 7 & 6 & 5 & 0.9524 \\
Indonesia & 79 & 10 & 8 & 0.6537 \\
Bangladesh & 98 & 12 & 24 & 0.6714 \\
China & 951 & 36 & 40 & 0.6336 \\
India & 4 & 4 & 2 & 1.0000 \\
Laos & 96 & 7 & 8 & 0.6583 \\
Nepal & 42 & 3 & 2 & 0.3821 \\
Taiwan & 29 & 3 & 6 & 0.1970 \\
Thailand & 54 & 9 & 10 & 0.7121 \\
Vietnam & 100 & 8 & 9 & 0.6541 \\
All & 1460 & 61 & 62 & 0.7020 \\
\hline
\end{tabular}


Table 3. Haplotype information based on sequence

\begin{tabular}{ll}
\hline Haplotype & Sequence \\
\hline Haplotype 1 & Syncerus caffer (D82888) \\
Haplotype 2 & Bubalus arnee (D32193) \\
Haplotype 3 & Bubalus mindorensis (D82895) \\
Haplotype 4 & Bubalus quarlesi (D82891) \\
Haplotype 5 & Bubalus depressicornis (D82890) \\
Haplotype 6 & JF946525, JF946524, EF409940, KR009082, KR009085-KR009088, KR009091-KR009093, KR009119, KR009125, KR009130, KR009133,
\end{tabular}

Haplotype 6 JF946525, JF946524, EF409940, KR009082, KR009085-KR009088, KR009091-KR009093, KR009119, KR009125, KR009130, KR009133, KR009135, KR009148, KR009155, KR009157, KR009159, KR009161, KR009162, KR009195, KR009207, KR009208, KR009233, KR009235, KR009238, KR009947, KR009948, KR009954, KR009965, KR009966, KR009972-KR009974, KR009985

Haplotype 7 JF946523

Haplotype 8 JF946522

Haplotype 9 JF946521

Haplotype 10 JF946520

Haplotype 11 JF946519

Haplotype 12 D82894, FJ467720, KR009887, KR009899, KR009900

Haplotype 13 BK6, BK7, BK8, BK9, BK14, BK15, BK21, BK24, BK28, BD1, BD4, BD7, BD9, BD11, BD13, BD14, BD15, BD19, BD25, KL4, KL5, KL6, KL9, KL10, KL11, KN2, KN11

Haplotype 14 BK12

Haplotype 15 BK18

Haplotype 16 KL1, KL2, KL7, KL8, KLB1, KLB2, KN8, KN9, KN10, KN13, TRB1, TRB2, TRB3, TRB4, TRB5, TRP6, TRP8, TRT13, TRT14, TRT15, NTB1, NTB2, NTB3, NTB5, NTB6, NTB7, NTB8, NTB9, NTB10, NTB11, BTN1, BTN2, BTN3, BTN4, BTN5, NAD1, NAD2, NAD3, FJ467715-FJ467719, FJ467721, FJ467725, FJ467727, FJ467729, FJ467761-FJ467779, FJ467781-FJ467917, KR009079, KR009081, KR009090, KR009094, KR009096, KR009099, KR009101, KR009103, KR009105, KR009106, KR009111, KR009167-KR009169, KR009172, KR009174, KR009176-KR009178, KR009181-KR009186, KR009192, KR009193, KR009202, KR009205, KR009214, KR009219, KR009223, KR009225, KR009226, KR009228, KR009229, KR009234, KR009243-KR009246, KR009249, KR009251-KR009254, KR009259, KR009262-KR009265, KR009267, KR009272-KR009274, KR009276, KR009277, KR009279, KR009280, KR009282, KR009285, KR009288, KR009290-KR009292, KR009295, KR009298, KR009303, KR009304, KR009307-KR009310, KR009314, KR009316, KR009318-KR009320, KR009324, KR009328KR009331, KR009333, KR009341-KR009346, KR009348, KR009350-KR009352, KR009356-KR009364, KR009366, KR009367, KR009369, KR009370-KR009372, KR009375, KR009376, KR009379, KR009381, KR009383-KR009387, KR009389, KR009391-KR009393, KR009396 KR009408, KR009410, KR009411, KR009416, KR009421, KR009424, KR009428-KR009431, KR009433, KR009435, KR009437-KR009440, KR009442-KR009455, KR009457, KR009459-KR009461, KR009463, KR009465-KR009480, KR009483-KR009486, KR009488, KR009491KR009498, KR009500-KR009502, KR009504-KR009507, KR009509-KR009511, KR009513, KR009515-KR009518, KR009520-KR009522, KR009524-KR009526, KR009528-KR009530, KR009532-KR009537, KR009539, KR009541-KR009550, KR009552, KR009554, KR009556KR009563, KR009565, KR009568-KR009571, KR009575, KR009579-KR009581, KR009583, KR009585-KR009587, KR009589-KR009594, KR009596-KR009599, KR009603-KR009605, KR009607, KR009609, KR009611, KR009612, KR009615-KR009618, KR009620, KR009622KR009624, KR009629, KR009632, KR009633, KR009636-KR009644, KR009688, KR009689, KR009692-KR009696, KR009698-KR009700, KR009702-KR009707, KR009710, KR009712, KR009714-KR009717, KR009719, KR009720, KR009723, KR009725, KR009726, KR009729, KR009730, KR009733, KR009736-KR009738, KR009740-KR009743, KR009745-KR009748, KR009750, KR009752, KR009754, KR009757, KR009758, KR009761, KR009762, KR009764, KR009765, KR009768-KR009770, KR009772, KR009773, KR009777, KR009780-KR009784, KR009786-KR009791, KR009793, KR009795-KR009798, KR009800, KR009801, KR009803, KR009804, KR009806, KR009807, KR009810, KR009812, KR009815, KR009818-KR009824, KR009826, KR009829-KR009831, KR009833, KR009834, KR009836, KR009838, KR009839, KR009842-KR009844, KR009847, KR009848, KR009850, KR009851, KR009856, KR009860, KR009861, KR009867, KR009868, KR009870, KR009871, KR009872, KR009874, KR009881, KR009884, KR009888-KR009890, KR009893, KR009897, KR009901-KR009904, KR009907, KR009911-KR009913, KR009922, KR009924-KR009929, KR009932-KR009937, KR009939-KR009941, KR009943, KR009986, KR009987, KR009990, KR009994-KR009996, KR009998, KR010000, KR010001, KR010004, KR010005, KR010007, KR010008, KR010012-KR010014, KR010018, KR010019, KR010021, KR010022, KR010027-KR010029, KR010030, KR010034, KR010039-KR010044, KR010046-KR010058, KR010060-KR010071, KR010073-KR010075, KR010079, KR010081, KR010086, KR010088, KR010090, KR010091, KR010094-KR010096, KR010098-KR010100, KR010102, KR010106, KR010108, KR010109, KR010112, KR010114-KR010116, KR010118, KR010121, KR010124KR010126, KR010128, KR010129, KR010133-KR010136, KR010139, KR010144-KR010149, KR010152-KR010154, KR010156-KR010159, KR010161, KR010163, KR010165, KR010168

Haplotype 17 KL3

Haplotype 18 KN1, KN4, KN6

Haplotype 19 KN3, KN5, KN7, KN12, KN14

Haplotype 20 NAD9

Haplotype 21 NAD10

Haplotype 22 FJ467648, EF409942, KR009200, KR009231, KR009650, KR009653, KR009667, KR009671, KR009955

Haplotype 23 FJ467649-FJ467657, EF409941, KR009069-KR009077, KR009080, KR009083, KR009084, KR009089, KR009102, KR009104, KR009107, KR009108, KR009112, KR009117, KR009118, KR009121-KR009124, KR009126, KR009127, KR009129-KR009132, KR009134, KR009136KR009139, KR009141-KR009144, KR009147, KR009149-KR009154, KR009156, KR009158, KR009160, KR009163, KR009164-KR009166, KR009194, KR009196, KR009198, KR009199, KR009201, KR009203, KR009204, KR009209, KR009210, KR009212, KR009215, KR009227, KR009230, KR009236, KR009239-KR009241, KR009645-KR009649, KR009651, KR009652, KR009654-KR009666, KR009668-KR009670, KR009672-KR009686, KR009944-KR009946, KR009949-KR009953, KR009956-KR009964, KR009967-KR009971, KR009975-KR009984

Haplotype 24 FJ467658, FJ467659, FJ467687-FJ467699, FJ467703-FJ467707, KR009170, KR009171, KR009175, KR009179, KR009180, KR009187KR009191, KR009206, KR009232, KR009296, KR009299 KR009301, KR009321, KR009353, KR009355, KR009365, KR009378, KR009380, KR009382, KR009388, KR009394, KR009412, KR009413, KR009478, KR009503, KR009508, KR009512, KR009514, KR009527, KR009538, KR009551, KR009555, KR009564,KR009566, KR009573, KR009606, KR009608, KR009621, KR009626, KR009627, KR009630, KR009635, KR009697, KR009728, KR009731, KR009732, KR009749, KR009771, KR009817, KR009823, KR009905, KR010032, KR010072, KR010101, KR010110, KR010117, KR010120, KR010123, KR010150 


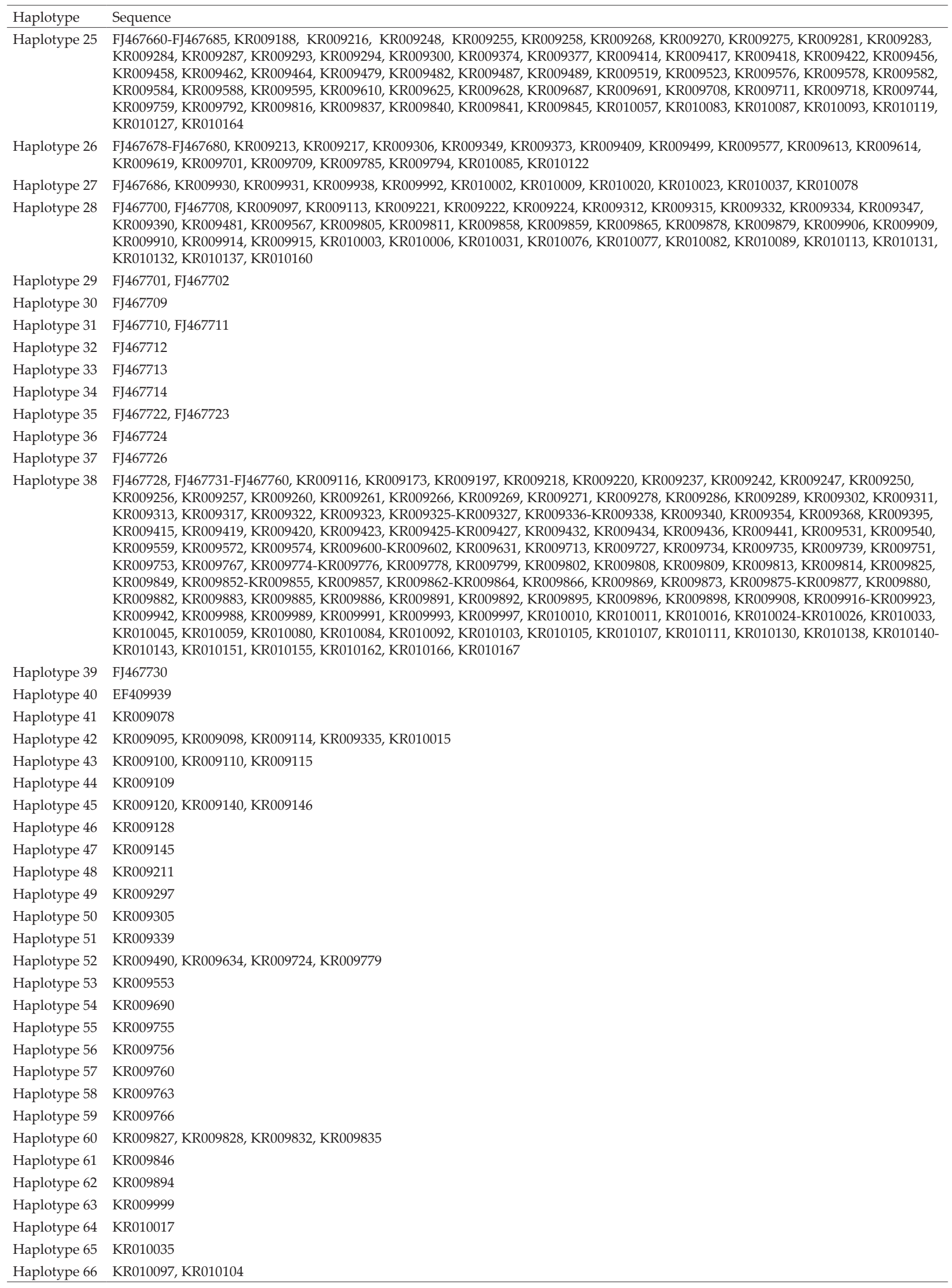


Table 4. Frequency of haplotype in ten countries

\begin{tabular}{|c|c|c|c|c|c|c|c|c|c|c|}
\hline \multirow{2}{*}{ Haplotype } & \multicolumn{10}{|c|}{ Countries } \\
\hline & Pakistan & Indonesia & Bangladesh & China & India & Laos & Nepal & Taiwan & Thailand & Vietnam \\
\hline 6 & $5.41 \%(2)$ & & $54.06 \%(20)$ & $13.51 \%$ (5) & $2.70 \%(1)$ & & $24.32 \%(9)$ & & & \\
\hline 7 & $100 \%(1)$ & & & & & & & & & \\
\hline 8 & $100 \%(1)$ & & & & & & & & & \\
\hline 9 & $100 \%(1)$ & & & & & & & & & \\
\hline 10 & $100 \%(1)$ & & & & & & & & & \\
\hline 11 & $100 \%(1)$ & & & & & & & & & \\
\hline 12 & & $20 \%(1)$ & & $20 \%(1)$ & & $60 \%(3)$ & & & & \\
\hline 13 & & $100 \%(27)$ & & & & & & & & \\
\hline 14 & & $100 \%(1)$ & & & & & & & & \\
\hline 15 & & $100 \%(1)$ & & & & & & & & \\
\hline 16 & & $5.05 \%(38)$ & $1.45 \%(11)$ & $73.40 \%(552)$ & & $5.85 \%(44)$ & & $3.47 \%(26)$ & $3.47 \%(26)$ & $7.31 \%(55)$ \\
\hline 17 & & $100 \%(1)$ & & & & & & & & \\
\hline 18 & & $100 \%(3)$ & & & & & & & & \\
\hline 19 & & $100 \%(5)$ & & & & & & & & \\
\hline 20 & & $100 \%(1)$ & & & & & & & & \\
\hline 21 & & $100 \%(1)$ & & & & & & & & \\
\hline 22 & & & & $87.5 \%(7)$ & $12.5 \%(1)$ & & & & & \\
\hline 23 & & & & $61.18 \%(52)$ & $1.18 \%(1)$ & & $37.64 \%(32)$ & & & \\
\hline 24 & & & & $88.89 \%$ (72) & & $1.23 \%(1)$ & & & $1.23 \%(1)$ & $8.65 \%(7)$ \\
\hline 25 & & & & $91.57 \%(76)$ & & $1.2 \%(1)$ & & & & $7.23 \%(6)$ \\
\hline 26 & & & & $90 \%(18)$ & & & & & & $10 \%(2)$ \\
\hline 27 & & & & $9.09 \%(1)$ & & $27.27 \%(3)$ & & & $54.55 \%(6)$ & $9.09 \%(1)$ \\
\hline 28 & & & $5.13 \%(2)$ & $38.46 \%$ (15) & & $25.64 \%(10)$ & & & $7.69 \%(3)$ & $23.08 \%$ (9) \\
\hline 29 & & & & $100 \%(2)$ & & & & & & \\
\hline 30 & & & & $100 \%$ & & & & & & \\
\hline 31 & & & & $100 \%(2)$ & & & & & & \\
\hline 32 & & & & $100 \%(1)$ & & & & & & \\
\hline 33 & & & & $100 \%(1)$ & & & & & & \\
\hline 34 & & & & $100 \%(1)$ & & & & & & \\
\hline 35 & & & & $100 \%(2)$ & & & & & & \\
\hline 36 & & & & $100 \%(1)$ & & & & & & \\
\hline 37 & & & & $100 \%(1)$ & & & & & & \\
\hline 38 & & & $0.58(1)$ & $60.82 \%(104)$ & & $19.88 \%(34)$ & & $1.17 \%(2)$ & $7.02 \%(12)$ & $10.53 \%(18)$ \\
\hline 39 & & & & $100 \%(1)$ & & & & & & \\
\hline 40 & & & & & $100 \%(1)$ & & & & & \\
\hline 41 & & & $100 \%(1)$ & & & & & & & \\
\hline 42 & & & $60 \%(3)$ & $20 \%(1)$ & & & & & $20 \%(1)$ & \\
\hline 43 & & & $100 \%$ & & & & & & & \\
\hline 44 & & & $100 \%$ (1) & & & & & & & \\
\hline 45 & & & $100 \%(3)$ & & & & & & & \\
\hline 46 & & & $100 \%(1)$ & & & & & & & \\
\hline 47 & & & $100 \%$ (1) & & & & & & & \\
\hline 48 & & & & $100 \%(1)$ & & & & & & \\
\hline 49 & & & & $100 \%(1)$ & & & & & & \\
\hline 50 & & & & $100 \%(1)$ & & & & & & \\
\hline 51 & & & & $100 \%$ (1) & & & & & & \\
\hline 52 & & & & $100 \%(4)$ & & & & & & \\
\hline 53 & & & & $100 \%(1)$ & & & & & & \\
\hline 54 & & & & $100 \%(1)$ & & & & & & \\
\hline 55 & & & & $100 \%(1)$ & & & & & & \\
\hline 56 & & & & $100 \%(1)$ & & & & & & \\
\hline 57 & & & & $100 \%(1)$ & & & & & & \\
\hline 58 & & & & $100 \%(1)$ & & & & & & \\
\hline 59 & & & & $100 \%(1)$ & & & & & & \\
\hline 60 & & & & $100 \%(4)$ & & & & & & \\
\hline 61 & & & & $100 \%(1)$ & & & & & & \\
\hline 62 & & & & & & $100 \%(1)$ & & & & \\
\hline 63 & & & & & & & & & $100 \%(1)$ & \\
\hline 64 & & & & & & & & & $100 \%(1)$ & \\
\hline 65 & & & & & & & & & $100 \%$ (1) & \\
\hline 66 & & & & & & & & & & $100 \%(2)$ \\
\hline
\end{tabular}




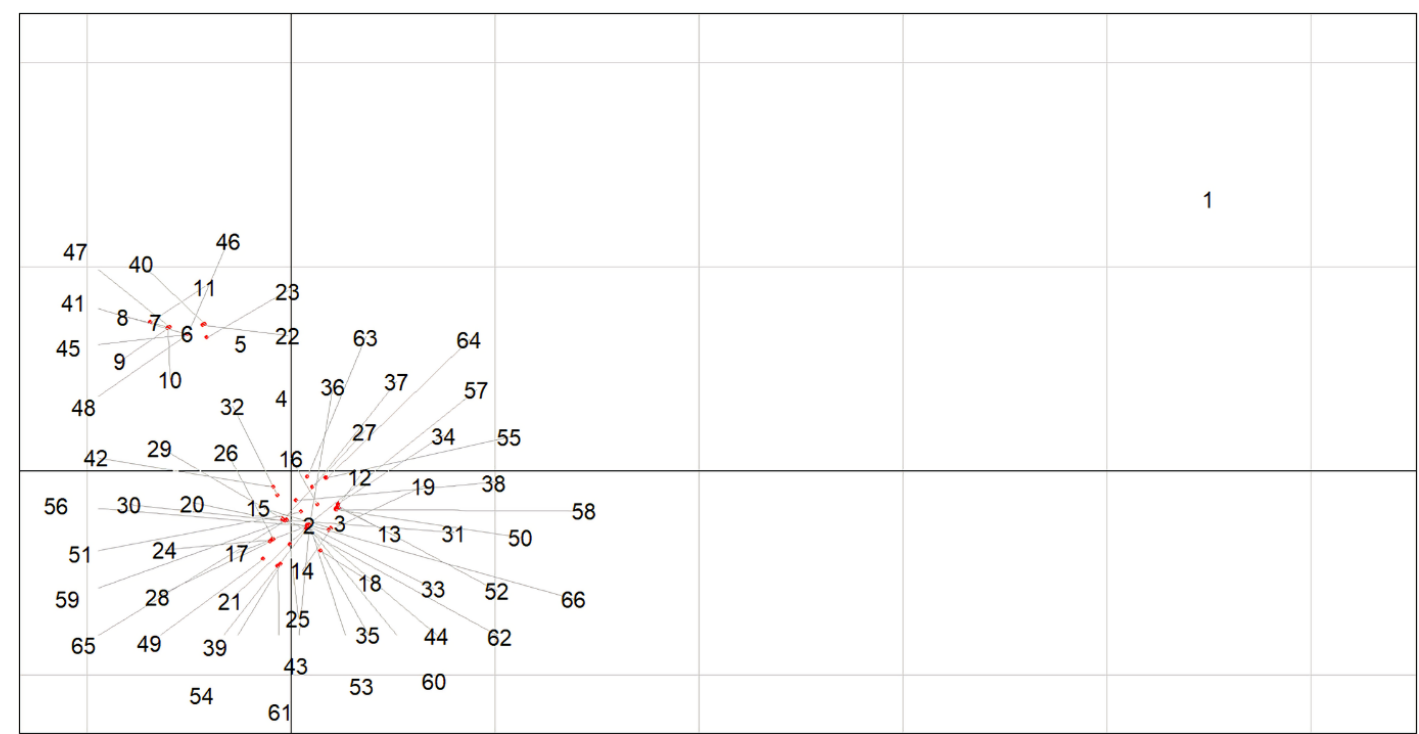

Figure 1. Principal coordinate analysis of haplotype based on cytochrome B

\section{Cluster plot}

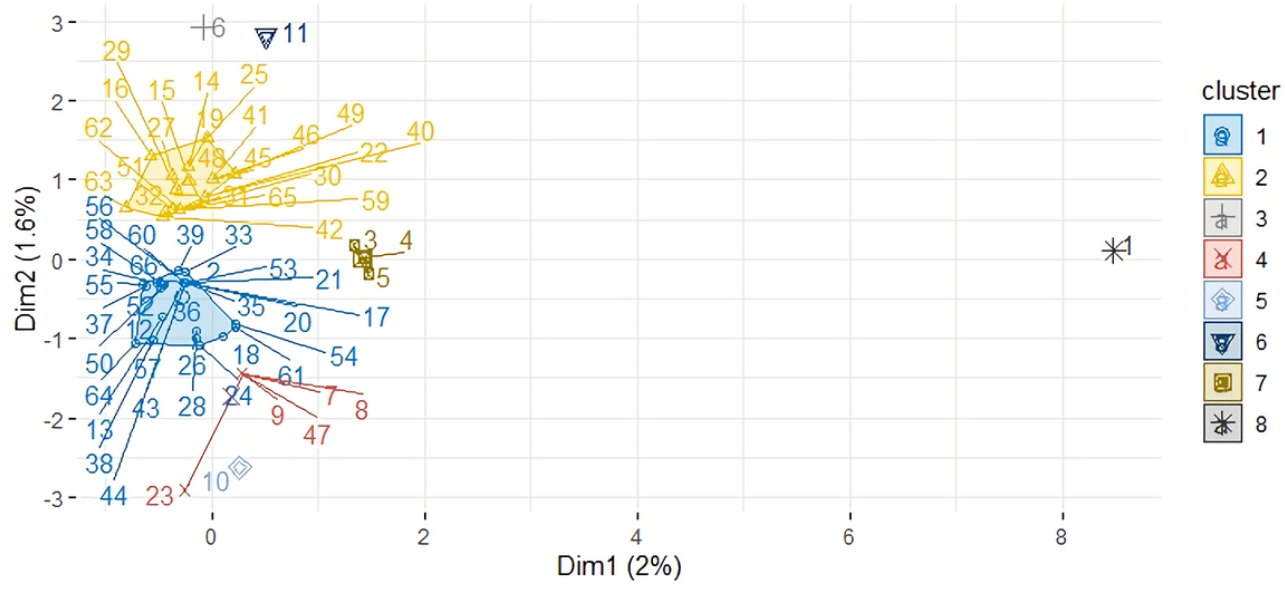

Figure 2. Hierarchical clustering on principal components of haplotype based on cytochrome B

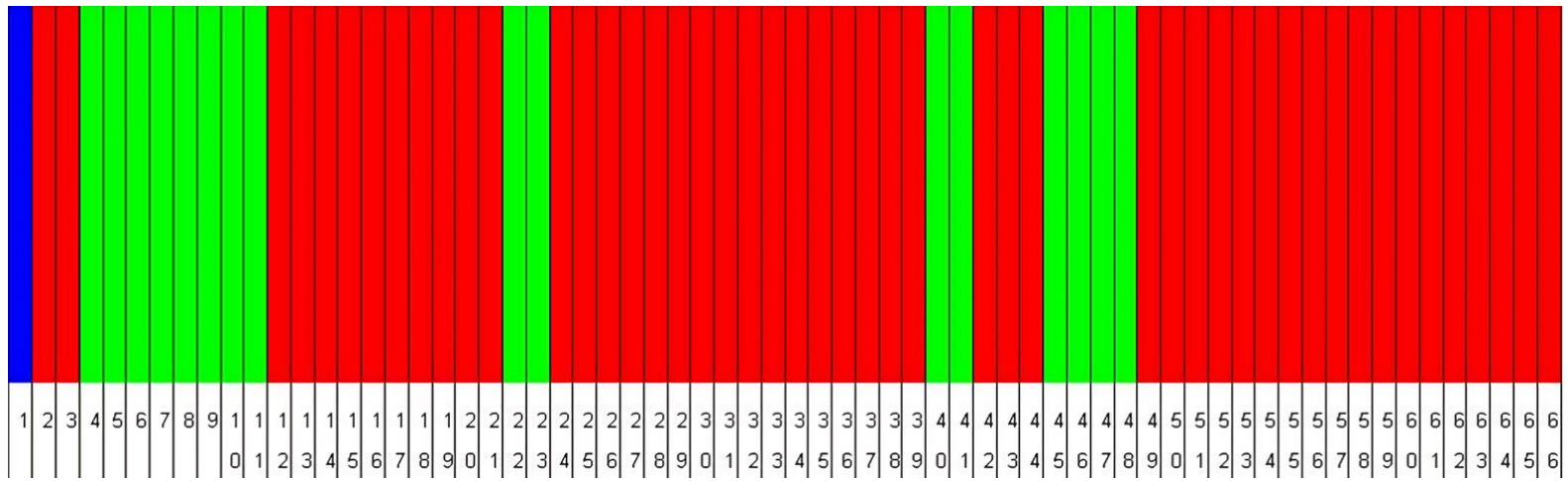

Figure 3. Bayesian analysis of cytochrome B gene haplotypes by BAPS software. Number represent haplotype and color represent cluster (blue= cluster 1 , red= cluster 2 , and green= cluster 3 ). 
words, the domestication of wild water buffalo occurred on the border of South and East Asia. Rusdin et al. (2020) found 10 haplotypes and 9 polymorphic sites for swamp buffalo in Indonesia. Based on this meta-analysis study, we found 8 unique haplotypes of Indonesian swamp buffalo.

Lei et al. (2007) found two maternal lineages in Chinese Native Swamp Buffaloes. We got different results because we added sequences carried out by Zhang et al. (2016) so that we found four maternal lineages of swamp buffalo in China and Bangladesh. Based on the complete mitochondrial genome of swamp buffalo revealed eight maternal lineages and this evidence support initial major domestication of swamp buffalo, probably between southern China and Vietnam (Wang et al., 2017). River and swamp buffaloes were domesticated independently based on these findings. Using the whole genome sequencing, Luo et al. (2020) found swamp and river buffaloes shared a common ancestor 1.1-3.5 million years ago. Based on the results of this meta-analysis, it is possible that the domestication of swamp buffalo occurred in East-South Asia.

\section{CONCLUSION}

Based on this study, we found four maternal lineages and unique haplotypes for swamp buffaloes, also two maternal lineages for river buffaloes as an outgroup in this study. Based on this research, we suggested that domestication of swamp buffalo occurred in East-South Asia, and domestication of river buffalo occurred in South Asia. Further research with a large sample size in Asian countries is needed to determine the exact number of maternal lineages. Meta-analysis can contribute to a good understanding of the overall data that has been studied to provide comprehensive conclusions.

\section{CONFLICT OF INTEREST}

Cece Sumantri serves as an editor of the Tropical Animal Science Journal, but has no role in the decision to publish this article. The authors declare that there is no conflict of interest with any financial, personal, or other relationships with other people or organizations related to the material discussed in the manuscript.

\section{REFERENCES}

Andreas, E., C. Sumantri, H. Nuraini, A. Farajallah, \& A. Anggraeni. 2010. Identification of GHIALUI and GHR I ALUI genes polymorphisms in Indonesian Buffalo. J. Indonesian Trop. Anim. Agric. 35:215-221. https://doi. org/10.14710/jitaa.35.4.215-221

Cheng, L., T. R. Connor, J. Sirén, D. M. Aanensen, \& J. Corander. 2013. Hierarchical and spatially explicit clustering of DNA sequences with BAPS software. Mol. Biol. Evol. 30:1224-1228. https://doi.org/10.1093/molbev/mst028

Chikuni, K., Y. Mori, T. Tabata, M. Saito, M. Monma, \& M. Kosugiyama. 1995. Molecular phylogeny based on the $\kappa$-casein and cytochrome $\mathrm{b}$ sequences in the mammalian suborder Ruminantia. J. Mol. Evol. 41:859-866. https://doi. org/10.1007/BF00173165

Guangxin, E., Z. Yong-Ju, N. Ri-Su, M. Yue-Hui, Z. Jia-Hua, C.
LiPeng, Q. Xiao-Yu, Z. Zhong-Quan, S. Ya-Wang, W. Xin, \& H. Yong-Fu. 2016. Meta-analysis evidence of maternal lineages in Chinese Tibetan sheep using mtDNA D-loop panel, Mitochondrial DNA Part A. https://doi.org/10.3109/ 24701394.2016.1143469

Husson, F., J. Josse, S. Le, \& J. Mazet. 2015. FactoMineR: Multivariate Exploratory Data Analysis and Data Mining. R Package Version 1.31.4. 24:1-18.

Jombart, T. 2008. Adegenet: A R package for the multivariate analysis of genetic markers. Bioinform. 11:1403-1405. https://doi.org/10.1093/bioinformatics/btn129

Kassambara, A., \& F. Mundt. 2020. factoextra: Extract and Visualize the Results of Multivariate Data Analyses ( $\mathrm{R}$ package version 1.0.7). https://cran.r-project.org/ package $=$ factoextra

Kumar, S., M. Nagarajan, J. S. Sandhu, N. Kumar, V. Behl, \& G. Nishanth. 2007. Mitochondrial DNA analyses of Indian water buffalo support a distinct genetic origin of river and swamp buffalo. Anim. Genet. 38:227-232. https://doi. org/10.1111/j.1365-2052.2007.01602.x

Kumar, S., G. Stecher, \& K. Tamura. 2016. MEGA7: Molecular evolutionary genetics analysis version 7.0 for bigger datasets. Mol. Biol. Evol. 33:1870-1874. https://doi.org/10.1093/ molbev/msw054

Lei, C. Z., C. M. Zhang, S. Weining, M. G. Campana, M. A. Bower, X. M. Zhang, L. Liu, X. Y. Lan, \& H. Chen. 2011. Genetic diversity of mitochondrial cytochrome $b$ gene in Chinese native buffalo. Anim. Genet. 42:432-436. https:// doi.org/10.1111/j.1365-2052.2011.02174.x

Lei, C. Z., W. Zhang, H. Chen, F. Lu, Q. L. Ge, R. Y. Liu, R. H. Dang, Y. Y. Yao, L. B. Yao, Z. F. Lu, \& Z. L. Zhao. 2007. Two maternal lineages revealed by mitochondrial DMA D-loop sequences in Chinese native water buffaloes (bubalus bubalis). Asian-Australas. J. Anim. Sci. 20:471-476. https://doi. org/10.5713/ajas.2007.471

Luo, X., Y. Zhou, B. Zhang, Y. Zhang, X. Wang, T. Feng, Z. Li, K. Cui, Z. Wang, C. Luo, H. Li , Y. Deng, F. Lu , J. Han, Y. Miao, H. Mao, X. Yi , C. Ai, S. Wu, A. Li, Z. Wu, Z. Zhuo, D. D. Giang, B. Mitra, M. F. Vahidi, S. Mansoor, S. Ahmed Al-Bayatti, Eka Meutia Sari, N. A. Gorkhali, S. Prastowo, L. Shafique, G. Ye, Q. Qian, B. Chen, D. Shi, J. Ruan, \& Q. Liu. 2020. Understanding divergent domestication traits from the whole-genome sequencing of swamp-and river-buffalo populations. Natl. Sci. Rev. 7:686-701. https://doi.org/10.1093/nsr/nwaa024

Mastrochirico-Filho, V. A., F. Pazo. M. E. Del Hata, G. V. Villanova, F. Foresti, M. Vera, P. Martínez, F. PortoForesti, \& D. T. Hashimoto. 2019. Assessing genetic diversity for a pre-breeding program in piaractus mesopotamicus by snps and ssrs. Genes. 10:668. https://doi.org/10.3390/ genes10090668

Misrianti, R., C. Sumantri, \& A. Farajallah. 2010. Polymorphism identification of pit1 gene in indonesian buffaloes (Bubalus bubalis) and holstein-friesian cows. Media Peternakan. 33:131-136. https://doi.org/10.5398/medpet.2010.33.3.131

Nagarajan, M., K. Nimisha, \& S. Kumar. 2015. Mitochondrial DNA variability of domestic River buffalo (Bubalus bubalis) populations: Genetic evidence for domestication of River buffalo in Indian subcontinent. Genome. Biol. Evol. 7:1252-1259. https://doi.org/10.1093/gbe/evv067

Paraguas, A. M., T. P. C. Cailipan, E. B. Flores, \& L. P. Villamor. 2018. Morphology and phylogeny of swamp buffaloes (Bubalus bubalis) in Calayan Island, Cagayan. Philipp. J. Vet. Anim. Sci. 44:59-67.

R Core, T. 2020. R: A Language and Environment for Statistical Computing. $\mathrm{R}$ Foundation for Statistical Computing. https://www.r-project.org/

Rozas, J., A. Ferrer-Mata, J. C. Sanchez-Del Barrio, S. Guirao-Rico, P. Librado, S. E. Ramos-Onsins, \& A. 
Sanchez-Gracia. 2017. DnaSP 6: DNA sequence polymorphism analysis of large data sets. Mol. Biol. Evol. 34:32993302. https://doi.org/10.1093/molbev/msx248

Rusdin, M., D. D. Solihin, A. Gunawan, C. Talib, \& C. Sumantri. 2020. Genetic variation of eight Indonesian swamp-buffalo populations based on cytochrome $b$ gene marker. Trop. Anim. Sci. J. 3:1-10. https://doi.org/10.5398/ tasj.2020.43.1.1

Saif, R., M. Wasim, \& M. E. Babar. 2012. Molecular phylogeny of Pakistani riverine buffalo based on genetic variability of Mitochondrial cytochrome b gene. Mol. Biol. Rep. 39:97079714. https://doi.org/10.1007/s11033-012-1835-1

Saputra, F., Jakaria, A. Anggraeni, \& C. Sumantri. 2020. Genetic diversity of Indonesian swamp buffalo based on microsatellite markers. Trop. Anim. Sci. J. 43:191-196. https://doi. org/10.5398/tasj.2020.43.3.191

Saputra, F., Jakaria, \& C. Sumantri. 2013. Genetic variation of mtDNA cytochrome oxidase subunit I (COI) in Local swamp buffaloes in Indonesia. Med. Pet. 36:165-170. https://doi.org/10.5398/medpet.2013.36.3.165

Sumantri, C., R. Diyono, A. Farajallah, A. Anggraeni, \& E. Andreas. 2010. Pemanfaatan famili gen hormon pertumbuhan (GH, GHR, GHRH, dan PIT-1) untuk mendeteksi keragaman genetik kerbau di Kabupaten Pandeglang dan Lebak Provinsi Banten. Jurnal Ilmu Ternak dan Veteriner 15:286-296.

Sun, T., S. Wang, Q. Hanif, N. Chen, H. Chen, \& C. Lei. 2020. Genetic diversity of mitochondrial cytochrome $\mathrm{b}$ gene in swamp buffalo. Anim. Genet. 51:977-981. https://doi. org/10.1111/age.12997

Taberlet, P., E. Coissac, J. Pansu, \& F. Pompanon. 2011 Conservation genetics of cattle, sheep, and goats. C. S. Biologies. 334:247-254. https://doi.org/10.1016/j. crvi.2010.12.007
Tanaka, K., C. D. Solis, J. S. Masangkay, K. I. Maeda, Y. Kawamoto, \& T. Namikawa. 1996. Phylogenetic relationship among all living species of the genus Bubalus based on DNA sequences of the cytochrome $b$ gene. Biochem. Genet. 34:443-452. https://doi.org/10.1007/BF00570125

Tobe, S. S., A. C. Kitchener, \& A. M. T. Linacre. 2010 Reconstructing mammalian phylogenies: A detailed comparison of the cytochrome $\mathrm{b}$ and cytochrome oxidase subunit i mitochondrial genes. PLoS ONE. 5:e14156. https:// doi.org/10.1371/journal.pone.0014156

Wang, S., N. Chen, M. R. Capodiferro, T. Zhang, H. Lancioni, H. Zhang, Y. Miao, V. Chanthakhoun, M. Wanapat, M. Yindee, Y. Zhang, H. Lu, L. Caporali, R. Dang, Y. Huang, X. Lan, M. Plath, H. Chen, J. A. Lenstra, A. Achilli \& C. Lei. 2017. Whole mitogenomes reveal the history of swamp buffalo: initially shaped by glacial periods and eventually modelled by domestication. Sci Rep 7:4708 (2017). https:// doi.org/10.1038/s41598-017-048

Youssef, N. A., M. Curaudeau, S. M. El Nahas, A. A. M. Hassan \& A. Hassanin. 2021. Haplotype diversity in the mitochondrial genome of the Egyptian river buffalo (Bubalus bubalis). Mitochondrial DNA B Resour. 6:145-147. https://doi.or $\mathrm{g} / 10.1080 / 23802359.2020 .1852622$

Zhang, Yi, Y. Lu, M. Yindee, K-Y. Li, H-Y. Kuo, Y-T. Ju, S. Ye, M. O. Faruque, Q. Li, Y. Wang, V. C. Cuong, L. D. Pham, B. Bouahom, B. Yang, X. Liang, Z. Cai, D. Vankan, W. Manatchaiworakul, N. Kowlim, S. Duangchantrasiri, W. Wajjwalku, B. Colenbrander, Y. Zhang, P. Beerli, J. A. Lenstra, \& J. F. S. Barker. 2016. Strong and stable geographic differentiation of swamp buffalo maternal and paternal lineages indicates domestication in the China/ Indochina border region. Mol. Ecol. 25:1530-1550. https:// doi.org/10.1111/mec.13518

Zhang, Y., L. Colli, \& J. S. F. Barker. 2020. Asian water buffalo: domestication, history and genetics. Anim. Genet. 51:177191. https://doi.org/10.1111/age.12911 